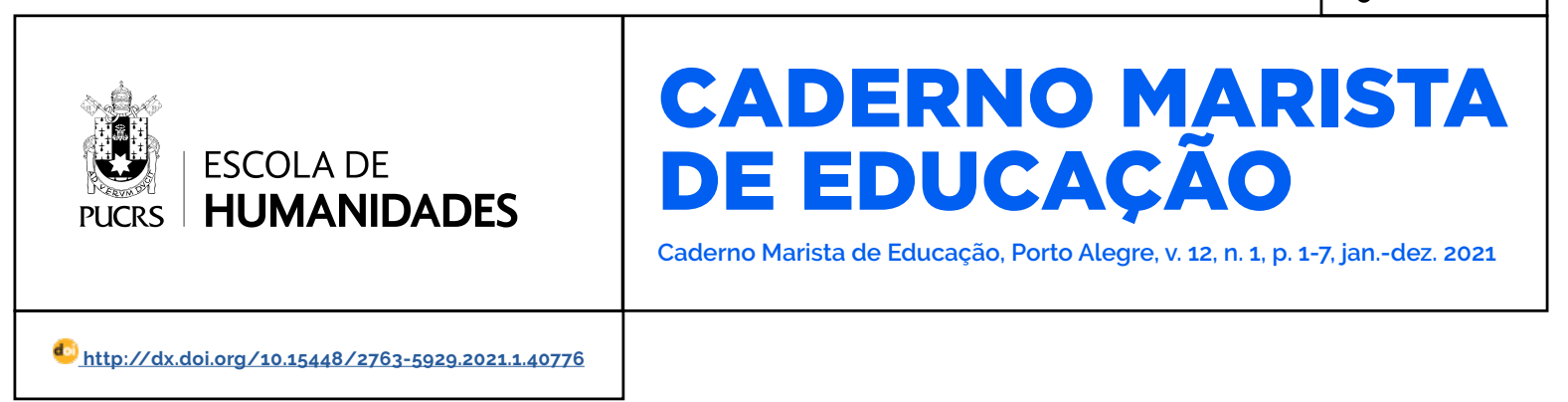

SEÇÃO: ARTIGOS

\title{
Calor ou temperatura? Uso de personagens da cultura geek para contextualização de fenômenos físicos/biológicos
}

\author{
Heat or temperature? use of geek culture characters for constextualization of physical \\ and biological phenomena
}

Artur Antunes Navarro

Valgas $^{1}$

orcid.org/0000-0002-8327-4562

artur.valgas@gmail.com

\section{Tatiane Alves \\ Gonçalves ${ }^{1}$}

orcid.org/0000-0001-6537-4968

tatianealves.goncalves@gmail.com

\section{Andrea Ferreira Pires da \\ Rosa $^{1}$}

orcid.org/0000-0003-1669-9461 andrea.rosa@maristas.org.br

Recebido em: 20 abr. 2021. Aprovado em: 24 ago. 2021. Publicado em: 05 out. 2021.
Resumo: A presente pesquisa buscou proporcionar a aprendizagem de conceitos básicos ligados a termorregulação, através do uso de personagens da cultura geek, a fim de tornar o conteúdo mais empático e próximo do cotidiano dos estudantes. Para tanto objetivou-se contextualizar fenômenos físicos e biológicos em duas turmas da primeira série do ensino médio por meio do uso de personagens da cultura geek em uma plataforma de gameficação. Para tanto, foi ministrada, de forma integrada entre os professores de física e biologia, uma aula sincrona através do Microsoft Teams, em que foram contextualizados os fenômenos físicos ligados à termorregulação. Com a finalidade de aproximar e aumentar o interesse dos estudantes, foi montado um painel interativo com personagens de animações, HQs e animes relacionados ao fogo e ao gelo. Cada personagem remetia a uma afirmação usada no dia a dia. Os estudantes deveriam justificar se a afirmação era verdadeira ou falsa na perspectiva da física. Com uso desse recurso, foi possivel observar a maior participação e empolgação dos estudantes, assim como uma maior assimilação dos conceitos trabalhados. Palavras-chave: Gameficação. Interdisciplinaridade. Juventudes. Ensino de ciências.

Absctract: The present research sought to provide the learning of basic concepts linked to thermoregulation, through the use of characters from the Geek culture, in order to make the content more empathic and closer to the daily lives of these students. To this end, the objective was to contextualize physical and biological phenomena in two classes of the first grade of high school through the use of characters from the geek culture on a gamification platform. For this purpose, a synchronous class was taught in an integrated way between the Physics and Biology components through Microsoft Teams, where the physical phenomena related to thermoregulation were contextualized. In order to bring students closer and increase their interest, an interactive panel with characters from animations, comics and animes, related to fire and ice, was set up. Each character referred to a statement used in everyday life, where students should justify whether the statement was true or false from the perspective of physics. Using this resource, it was possible to observe the greater participation and excitement of the students, as well as a greater assimilation of the concepts worked on.

Keywords: Gamefication. Interdisciplinarity. Youths. Science teaching.

\section{Introdução}

A pandemia causada pela COVID-19 mudou o cenário educativo, a sala de aula passou de um formato presencial para o virtual, a partir de transmissões em tempo real por meio de plataformas como o Microsoft Teams (MARTIN; TAPP, 2019). Para Fernandes (2021), atualmente, há possibilidade de exploração de ferramentas virtuais diversificadas com o avanço das tecnologias de informação e comunicação (TICs).
Artigo está licenciado sob forma de uma licenç Creative Commons Atribuição 4.0 Internacional. 
Entretanto, o autor salienta que muitas pesquisas educacionais concluem que os jovens se mostram insatisfeitos com métodos tradicionais de ensino, pois há diversas possibilidades mais atrativas para a juventude.

Os personagens de histórias em quadrinhos e animações fizeram e fazem parte do imaginário de crianças e adolescente em todo o mundo (DUARTE, 2018). Para muitos constituem parte importante da memória afetiva dessa fase do desenvolvimento humano (VIEIRA, 2019; D'ALTOÉ et al., 2020). Com heróis, vilões, enredos e histórias instigantes, as animações e quadrinhos envolvem e prendem a atenção do jovem, constituindo parte integrante da sua identidade sociocultural emocional de seu cotidiano (CANÁRIO, 2012; MENEZES, 2017; PEREIRA et al., 2020).

A cultura geek/nerd, segundo alguns autores (BICCA et al., 2013; MANSO, 2020; QUINTAIROS-SOLIÑO, 2019; SOUZA; LEITE, 2018; VARGAS; LOPES, 2020; NASCIMENTO, 2019; VILAR, 2015; NETO et al., 2020) configura-se como um grupo cultural da população que abrange individuos que apreciam tecnologias, aquisição de conhecimento (acadêmico ou não) e principalmente entretenimento/diversão através de filmes de ficção, seriados e vídeo games, jogos digitais, animações japonesas e norte-americanas.

O fim da década de 1990 é marcado pela aceitação das histórias em quadrinho (HQ) como estratégia no ensino no Brasil. Segundo Santos e Vergueiro (2012), esse fato está diretamente relacionado com a Lei de Diretrizes e Bases da Educação Nacional (LDB), que abriu espaço à inserção de novas linguagens para o processo de aprendizagem. Desde então, os educadores têm utilizado animes e histórias em quadrinhos (HQ) como ferramentas educacionais (SANTOS et al., 2019; SILVA; MESSIAS, 2019). O uso de personagens da cultura geek aproxima a sala de aula das vivências dos estudantes, agindo como facilitador do processo de aprendizagem (SANTOS, 2019).
Dentro desta perspectiva a seguinte pergunta foi feita: "Como fazer uso dos personagens da cultura geek para facilitar o aprendizado de conceitos ligados à biologia e a física no ensino remoto?". Objetivou-se com este trabalho contextualizar fenômenos físicos e biológicos em duas turmas da primeira série do ensino médio através do uso de personagens da cultura geek em uma plataforma em formato de jogo. A prática docente com o suporte de jogos, para Fernandes (2021), é um fator determinante no engajamento dos estudantes dos envolvidos no processo de aprendizagem.

\section{Desenvolvimento}

Um estudo considerado qualitativo possibilita a investigação dos fenômenos que envolvem os seres humanos e suas relações sociais em ambientes diversos (GODOY, 1995). Como define Gil (2008), para que haja uma pesquisa-ação é necessário a interação dos pesquisadores e membros do contexto investigado. Como os educadores autores desse trabalho estão envolvidos no processo de modo colaborativo essa pesquisa se desenvolveu em forma de um relato de experiência.

O desenvolvimento desse estudo qualitativo ocorreu durante uma aula integrada de 100 minutos (correspondente a 2 periodos) entre os componentes de física e biologia na temática de termorregulação, com o uso da plataforma on-line Microsoft Teams, onde ambos os docentes ingressaram na live ao mesmo tempo. O grupo de participantes é composto por duas turmas de primeira série do ensino médio do Colégio Marista São Pedro no ano de 2020, totalizando 40 estudantes.

Como forma introdutória, com a finalidade de retomar conteúdo chave para o entendimento da termorregulação, foi construido um painel interativo no Microsoft Powerpoint com a utilização de links. Esses links que direcionavam para alguns para os personagens da cultura geek ao clicar em cima do mesmo. 
Figura 1 - Painel Interativo
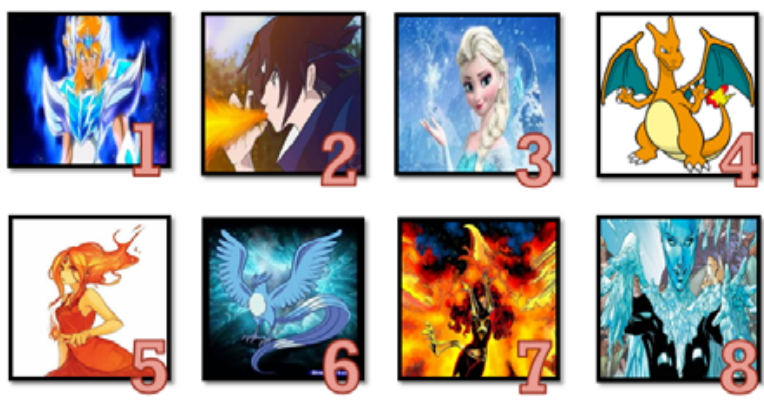

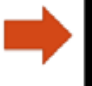

ESTOU COM CHLOR HOJE!
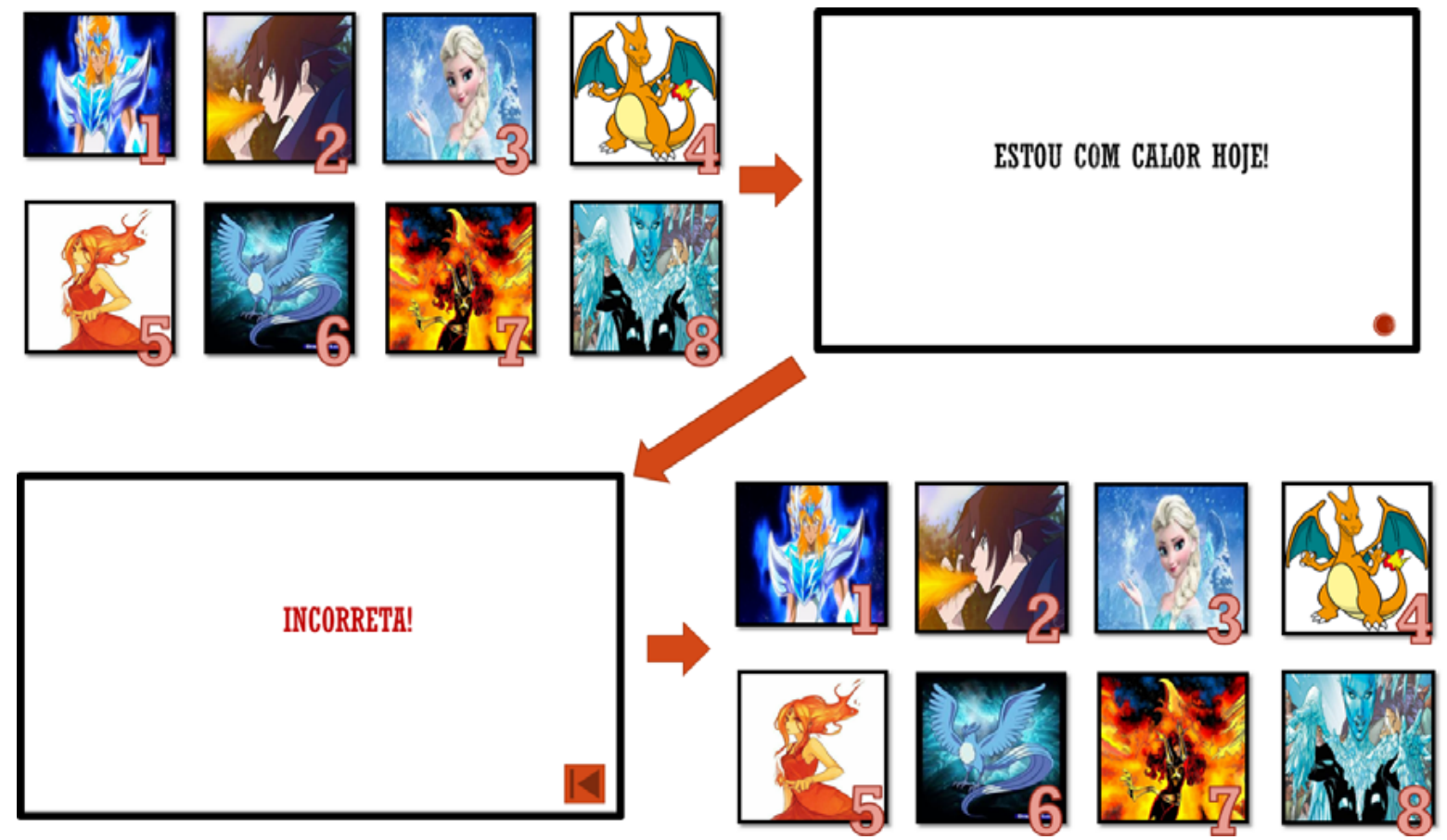

Fonte: Elaborado pelos autores (2021)

Legenda: Foram utilizados os personagens 1 = Hyoga (anime/mangá Cavaleiros do Zodíaco); 2 = Sasuke (anime/mangá Naruto); 3 = Elsa (animação Frozen); 4 = Charizard (anime/mangá Pokemon); 5 = Princesa de Fogo (animação Hora de Aventura); 6 = Articuno (anime/mangá Pokemon); 7 = Fenix (anime/HQ X-men); 8 = Nevasca (animação/HQ Liga da Justiça).

A dinâmica foi realizada da seguinte forma: Os estudantes escolhiam um número/personagem e eram direcionados para um slide, neste havia uma frase que é utilizada no cotidiano, como representado na Figura 2. Fazendo uso dos conhecimentos previamente trabalhados sobre calor e temperatura os estudantes tiveram que justificar se a afirmação era verdadeira ou falsa do ponto de vista físico, e após a decisão era revelado se a afirmação era verdadeira ou falsa (Figura 1). O que facilitou na consolidação do conhecimento acerca dos conceitos físicos de calor e temperatura, troca de calor/energia, que são importantes para o entendimento dos fenômenos relacionados a termobiologia. 
$4 / 7$ Caderno Marista de Educação, Porto Alegre, v. 12, n. 1, p. 1-7, jan.-dez. 2021 | e-40776

Figura 2 - Frases remetidas
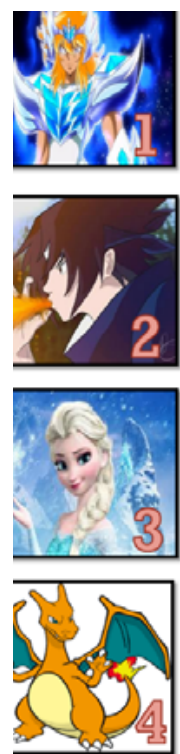
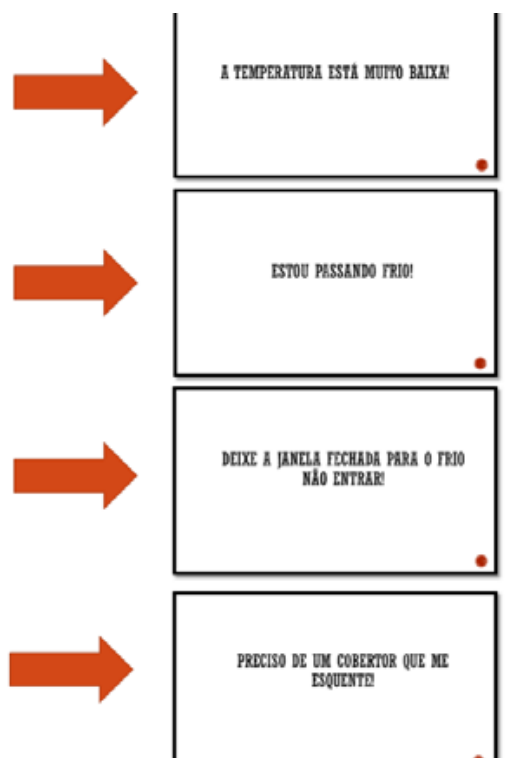
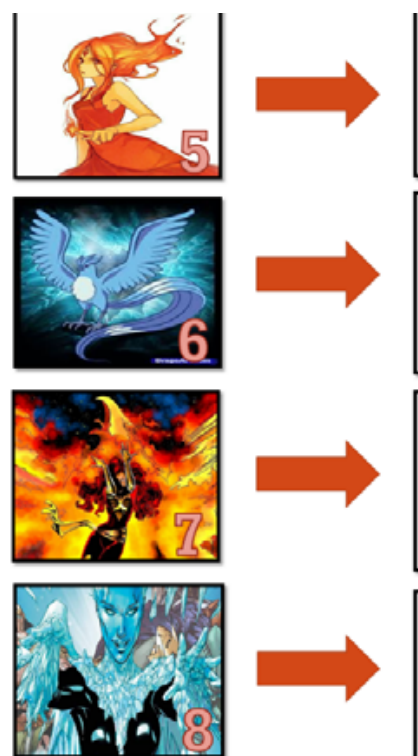

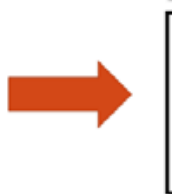

A TRIPRRATURA LSTh ULT LA TORA
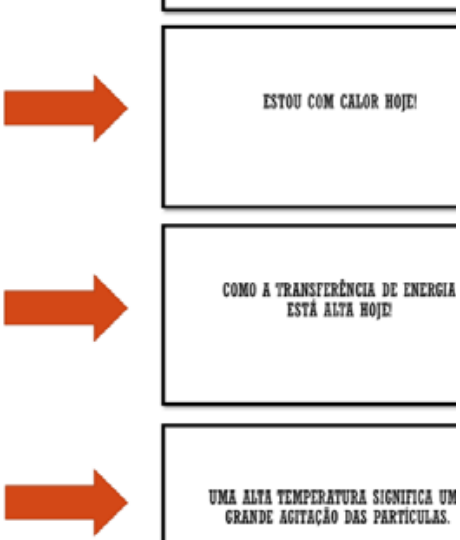

Fonte: Elaborado pelos autores (2021).

Legenda: Frases direcionadas: 1 - A temperatura está muito baixa!; 2 - Estou passando frio!; 3 - Deixe a janela fechada para o frio não entrar!; 4 - Preciso de um cobertor que me esquente!; 5 - A temperatura está alta lá fora!; 6 - Estou com calor hoje!; 7 - Como a transferência de energia está alta hoje!; 8 - Uma alta temperatura significa uma grande agitação das partículas.

Em aulas anteriores, os docentes de física e biologia perceberam uma grande afinidade das turmas com relação aos personagens, o que foi um fator determinante para o planejamento da atividade. Para Freire (2000), um dos aspectos para o bom desenvolvimento da aprendizagem dos estudantes é a interação, se a exposição do conteúdo acontecer de forma mais atrativa facilitará a compreensão. Na Figura 3 é possivel visualizar como a aula foi conduzida pelo Microsoft Teams. Para docentes e colaboradores da Rede Marista a gravação desta aula está disponivel na plataforma Microsoft Stream no Canal Ciências Biológicas São Pedro, Título Calor e Energia -Termorregulação, postado no dia 12 de agosto de 2020.

Figura 3 - Foto da execução da aula

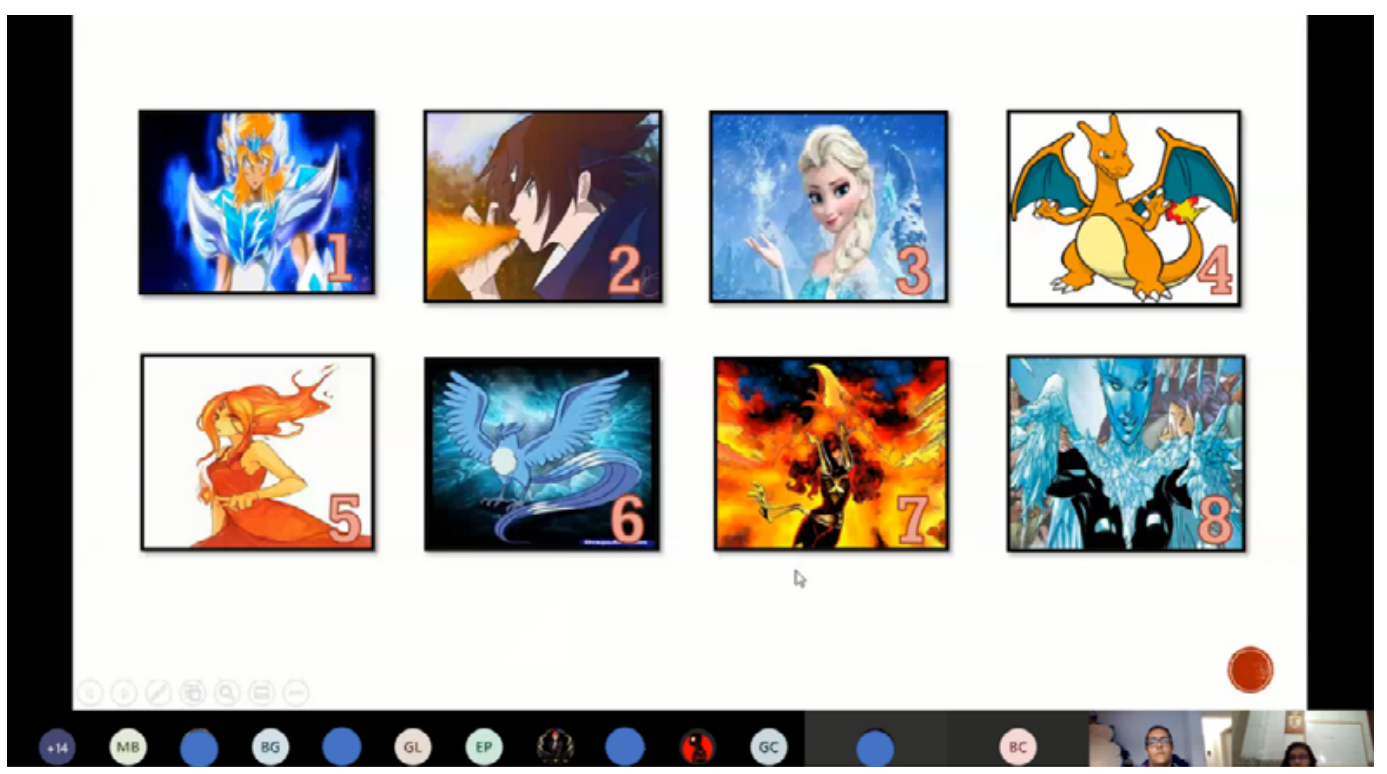

Fonte: Elaborado pelos autores (2021). 


\section{Resultados e discussão}

Trabalhar com a cultura geek é desafiante e instigante. Abordagens semelhantes podem ter um papel considerável no processo educativo segundo Santos e Vergueiro (2012). Foi possível perceber a efetividade na aprendizagem na primeira série do ensino médio, por meio do relato dos estudantes que afirmaram que os personagens são facilitadores do processo de aprendizagem por tornar o conteúdo mais atrativo e interessante (VALGAS; GONÇALVES; AMARAL, 2020). A aula integrada possibilitou aos estudantes o desenvolvimento das seguintes habilidades presentes na Base Nacional Comum Curricular (BNCC) (BRASIL, 2018, p. 559):

(EM13CNT305) Investigar e discutir o uso indevido de conhecimentos das Ciências da Natureza na justificativa de processos de discriminação, segregação e privação de direitos individuais e coletivos para promover a equidade e o respeito à diversidade;(EM13CNT306) Avaliar os riscos envolvidos em atividades cotidianas, aplicando conhecimentos das Ciências da Natureza, para justificar o uso de equipamentos e comportamentos de segurança, visando à integridade fisica, individual e coletiva, e socioambiental. (EM13CNT301) Construir questões, elaborar hipóteses, previsões e estimativas, empregar instrumentos de medição e representar e interpretar modelos explicativos, dados e/ ou resultados experimentais para construir, avaliar e justificar conclusões no enfrentamento de situações-problema sob uma perspectiva científica.

Dentro da área de ciências da natureza da BNCC, além das habilidades, identifica-se a competência 3, que é:

Investigar situações-problema e avaliar aplicações do conhecimento científico e tecnológico e suas implicações no mundo, utilizando procedimentos e linguagens próprios das Ciências da Natureza, para propor soluções que considerem demandas locais, regionais e/ou globais, e comunicar suas descobertas e conclusões a públicos variados, em diversos contextos e por meio de diferentes midias e tecnologias digitais de informação e comunicação (TDIC) (BRASIL, 2018, p. 553).

A ciência tem sua linguagem e quando seus conhecimentos são apropriados é possivel reconhecê-los no cotidiano e aplicá-los. Durante a atividade os estudantes foram questionados sobre alguns termos utilizados no dia a dia de forma incorreta, sendo assim, percebe-se uma relação direta com as competências de ciências (UNIÃO MARISTA DO BRASIL, 2019, p. 54): "apropriar-se de conhecimentos das ciências e entendê-los como instrumento de leitura do mundo." e "elaborar suposições e hipóteses sobre fenômenos estudados e cotejá-las com explicações científicas [...]". Ao responder aos questionamentos propostos, explorou-se a capacidade de trabalhar também a competência "elaborar comunicações orais ou escritas pertinentes a fenômenos e processos naturais, utilizando múltiplas linguagens e representações cientificas" (UNIÃO MARISTA DO BRASIL, 2019, p. 54).

Nas especificidades das competências acadêmicas da física foram identificadas, no transcorrer da atividade, "aplicar, em dada situação-problema, as informações ou variáveis relevantes e possiveis estratégias para resolvê-la" (UNIÃO MARISTA DO BRASIL, 2019, p. 100), além de "interpretar e propor modelos explicativos para fenômenos ou sistemas físicos ou tecnológicos" (UNIÃO MARISTA DO BRASIL, 2019, p. 100). Quando em aula é possivel trazer os conceitos presentes no dia a dia, relaciona-se a capacidade de "interpretar, compreender e discutir relações entre a ciência, a tecnologia, o ambiente e a sociedade e "mobilizar conhecimentos para emitir julgamentos e tomar posições a respeito de situações e problemas de interesse pessoal e social relativos às interações da ciência na sociedade." (UNIÃO MARISTA DO BRASIL, 2019, p. 40).

Conforme observado por diversos autores (BICCA et al., 2013: MANSO, 2020, 2019; SOUZA; LEITE, 2018; VARGAS; LOPES, 2020; NASCIMENTO, 2019; VILAR, 2015; NETO, et al., 2020; SILVA et al., 2019; SANTOS; SOUZA, 2019) o uso de personagens da cultura geek é um facilitador do processo de ensino e aprendizagem, uma vez que se tornam o conteúdo mais atrativo e relacionado ao cotidiano estudantil. Este fato foi observado durante a aplicação desta prática educativa, onde os estudantes apresentaram maior engajamento e empolgação com a atividade. Segundo Ramos et al. (2016), há a formação de aprendizado significativo quando são evocadas áreas do cérebro 
ligadas ao sistema límbico das emoções, ou seja, uma vez que o cérebro se emociona com algo há formação de memória de longo prazo e aprendizado. Silva et al. (2019) destaca:

A utilização de elementos de cultura popular em sala de aula, principalmente relacionados ao cotidiano do aluno, tende a atrair a atenção dos estudantes, contribuindo para uma aula mais dinâmica e facilitando o processo de aprendizado.

A afirmação vai ao encontro do observado em nossa prática docente, em que os estudantes apresentaram maior empolgação e maior participação na aula quando lançasse mão desses tópicos se comparado a temas trabalhados em momentos anteriores.

\section{Considerações finais}

O uso dos personagens na aula integrada teve maior aceitação pelas turmas, desse modo, podemos afirmar que os educadores precisam estar atentos às necessidades e interesses dos estudantes para tornar o processo de aprendizagem mais atrativo e instigante. $\mathrm{O}$ uso de personagens da cultura geek está ligado diretamente ao cotidiano estudantil, mostrando-se uma ferramenta importante no processo de ensino, gerando um aprendizado significativo, que será internalizado nas áreas de assimilação de memória de longo prazo.

Visto isto, acreditamos que um diálogo descontraido junto a corpo de estudantes, que permita ao professor conhecer o seu aluno, os seus gostos pessoais e a sua realidade, é fundamental para o processo de ensino e aprendizagem, pois o professor poderá detectar características da turma e traçar estratégias de ensino atrativas para aquele grupo de estudantes. Uma vez que os estudos na área de neurociência e educação têm cada vez mais apontado que uma aprendizagem significativa deve encantar e emocionar o cérebro, precisamos despertar o interesse, a curiosidade, a inquietação e as incertezas nos nossos estudantes. Uma forma de fazer isso é tornando o processo de ensino mais próximo dos interesses dos discentes.

\section{Referências}

BICCA, Angela Dillmann Nunes et al. Identidades Nerd/ Geek na web: um estudo sobre pedagogias culturais e culturas juvenis//Nerd/Geek Identities on the web: a study on cultural pedagogies and youth culture. CONJECTURA: filosofia e educação, Caxias do Sul, v. 18, n. 1, p. 87-104, 2013.

BRASIL. Ministério da Educação. Base Nacional Curricular Comum, 2018. [S. I.]: Ministério da Educação, 2018. Disponivel em: http://basenacionalcomum.mec. gov.br/images/historico/BNCC_EnsinoMedio_embaixa_site_110518.pdf. Acesso em: 20 abr. 2020.

CANÁRIO, Tiago. Mangás, animes, juventude e socialização: um estudo sobre o Efeito de Terceira Pessoal Mangas, animes, youth and socialization: a third-person effect study. Liinc em revista, Rio de Janeiro, v. 8, n. 2 , p. 493-508, 2012.

D'ALTOÉ, Francisca; JÚNIOR, Mario Abel Bressan. Economia e memória afetiva no cinema live-action: "Aladdin" e "O Rei Leão". Trama: indústria criativa em revista, Rio de Janeiro, v. 9, n. 1, p. 1-89, 2020. ISSN 2447-7516.

DUARTE, Renan Silva. Quadrinhos é coisa de criança: Considerações sobre um equivoco desimportante. Literartes, São Paulo, v. 1, n. 8, p. 208-220, 2018.

FERNANDES, Carlos Wilson Ribeiro. Aprendizagem em jogo: estudo e desenvolvimento conceitual de recursos digitais para apoio à alfabetização. In:

DICKMANN, Ivanio (org.). Start: como a gameficação e os jogos de aprendizagem estão transformando a práxis educativa atual com suas dinâmicas inovadoras e criativas. 1. ed. Chapecó: Livrologia, 2021. p. 89-110.

FREIRE, Flávio. A interação professor-aluno e suas implicações pedagógicas. UNOPAR Cient., Ciênc. Hum. Educ., Londrina, v. 1, n. 1, p. 115-121, jun. 2000.

GIL, Antônio Carlos. Métodos e técnicas de pesquisa social. 6. ed. São Paulo: Atlas, 2008.

GODOY, Arllda Schmidt. Pesquisa qualitativa: tipos fundamentais. Revista de Administração de empresas, São Paulo, v. 35, n. 3, p. 20-29. maio/jun., 1995.

LOPES, Arian Júnior dos Santos; OLIVEIRA, Carlos Allan de Souza. Utilização do anime Pokémon para o ensino-aprendizagem de ciências naturais. In: CONGRESSO NACIONAL DE PESQUISA E ENSINO EM CIÊNCIAS E TECNOLOGIA, INVESTIGAÇÃO, SUSTENTABILIDADE E OS DESAFIOS DO SÉCULO XXI, 4., 2019, Campina Grande. Anais [...]. Campina Grande: Realize Editora, 2019. Disponivel em: https://editorarealize.com.br/ artigo/visualizar/57324. Acesso em: 24 set. 2021.

MANSO, Cátia Daniela Pinheiro. Perfil do consumidor Nerd: um exemplo de subcultura urbana. 2020. Dissertação (Mestrado em Marketing e Negócios Internacionais) - Instituto Superior de Contabilidade e Administração de Coimbra, Coimbra, jul. 2020.

MARTIN, Louis; TAPP, Dave. Teaching with Teams: An introduction to teaching an undergraduate law module using Microsoft Teams. Innovative Practice in Higher Education, Staffordshire, v. 3, n. 3, p. 58-66, 2019. 
MENEZES, Érika Lourenço de. Heróis e heroinas ou apenas colegiais: juventude e os desenhos animados. 2017. Dissertação (Mestrado em Educação) - Universidade Federal do Estado do Rio De Janeiro, Rio de Janeiro, 2017.

MONTALVÃO NETO, Alberto Lopo; NAKAMURA, Tayna Mioni; DA SILVA, Francisco Vieira. Representações da ciência e da mulher cientista na série animada "Hora de Aventura". Palimpsesto-Revista do Programa de Pós-Graduação em Letras da UERJ, Rio de Janeiro, v. 19, n. 32, p. 142-158, 2020.

NASCIMENTO, Francisco. Stan Lee, o Quarteto Fantástico e a evolução da divulgação científica nas histórias em quadrinhos de super-heróis: possibilidades para uma aula de ciências. Diálogo, Canoas, n. 42, p. 55-65, 2019.

PEREIRA, Gabriela Pereira de; FERREIRA, Maira. Matemática, Arte e Mangá: a cultura audiovisual em diálogo com as juventudes. Zetetike, Campinas, v. 28, p. 1-19, 2020. e020032.

QUINTAIROS-SOLIÑO, Alba. Contemporary Japanese Folktales Represented in Anime: the Paradigmatic Case of InuYasha. In: BRUGUÉ, Lidia; LLOMPART, Auba Contemporary Fairy-Tale Magic. Leiden: Brill Rodopi, 2019. p. 273-285.

RAMOS, Daniela Karine; LORENSET, Caroline Chioquetta; PETRI, Giani. Jogos educacionais: contribuições da neurociência à aprendizagem. Revista X, Curitiba, v. 2 , n. 1, p. 1-17, 2016

SANTOS, Roberto Elísio dos; VERGUEIRO, Waldomiro. Histórias em quadrinhos no processo de aprendizado: da teoria à prática. Eccos Revista Científica, São Paulo, n. 27, p. 81-95, 2012

SANTOS, Romário da Silva. Cultura geek e construção de redes de sociabilidade e saberes: um estudo sobre protagonismos juvenis em Horizonte-Ce. 2019. Dissertação (Mestrado em Sociologia) - Universidade Federal do Ceará, Fortaleza, 2019.

SILVA, Kaique Cesar de Paula; MESSIAS, Thiago Silva. Cultura pop, o uso de pokémon como ferramenta de ensino das ciências biológicas. Cultura pop, o uso de pokémon como ferramenta de ensino das ciências biológicas. In: DALAZOANA, Karine (org.). Fundamentos e aplicações da Biologia. Ponta Grossa: Atena Editora, 2019. p. 388-416. Disponivel em: https://sistema.atenaeditora.com.br/index.php/admin/api/artigoPDF/15610. Acesso em: 20 abr. 2020.

SOUZA, Jéssica Itaiane R. de; LEITE, Bruno S. Utilização das Séries de TV no Ensino de Química. Rev. Virtual Química, Niterói, v. 10, n. 4, p. 749-766, 2018.

UNIÃO MARISTA DO BRASIL. Matrizes curriculares de educação básica do Brasil Marista: área de ciências da natureza e suas tecnologias. 3. ed. Curitiba: PUCPRESS, 2019.

VALGAS, Artur Antunes Navarro; GONÇALVES, Tatiane ALves; AMARAL, Lisandra do Catalan. Biofísica: Integrando os componentes de Biologia e a Física no Ensino Remoto. Revista de Estudos e Pesquisas sobre Ensino Tecnológico (EDUCITEC), Manaus, v. 6, p. 1-16, e155820, 2020.

VARGAS, João Carlos Martini de; LOPES, Leticia Azambuja. O ensino de ciências e a franquia star wars: possibilidades pedagógicas. e-Mosaicos, Rio de Janeiro, v. 9. n. 22, p. 236-250, 2020.
VIEIRA, Nicole Pachêco. O uso de animações japonesas na educação: protagonismo infantil, imaginação e empatia. 2019. Trabalho de Conclusão de Curso (Graduação em Educação) - Universidade de Brasilia, Brasilia, DF, 2019.

VILAR, Fabiolla Emanuelle Silva. Conectando-se a Perfect World: notas preliminares acerca da sociabilidade em um espaço social digital. Revista Elaborar. Manaus, v. 2, n. 1, p. 140-163, 2015

\section{Artur Antunes Navarro Valgas}

Mestre em Biologia Animal pela UFRGS. Bacharel e Licenciado em Ciências Biológicas pela Pontificia Universidade Católica do Rio Grande de Sul (PUCRS), em Porto Alegre, RS, Brasil. Doutorando no Programa de Pós-Graduação em Biologia Animal da Universidade Federal do Rio Grande do Sul (UFRGS), em Porto Alegre, RS, Brasil. Professor de Ciências e Biologia no Colégio Marista São Pedro, em Porto Alegre, RS, Brasil.

\section{Tatiane Alves Gonçalves}

Mestre em Educação em Ciências pela Pontificia Universidade Católica do Rio Grande do Sul (PUCRS), em Porto Alegre, RS, Brasil. Licenciada em Física pela Pontifícia Universidade Católica do Rio Grande do Sul (PUCRS), em Porto Alegre, RS, Brasil. Doutoranda no Programa de Pós-Graduação em Ensino de Física da Universidade Federal do Rio Grande do Sul (UFRGS), em Porto Alegre, RS, Brasil. Professora da Escola Técnica Mascarenhas de Moraes, em Cachoeirinha, RS, Brasil, e no Colégio Marista São Pedro, em Porto Alegre, RS, Brasil.

\section{Andrea Ferreira Pires da Rosa}

Especialista em Educação Inclusiva pela Pontificia Universidade Católica do Rio Grande do Sul (PUCRS), em Porto Alegre, RS, Brasil. Especialista em Supervisão Educacional pelo Centro Universitário Leonardo da Vinci, em Novo Hamburgo, RS, Brasil. Pedagoga pela Pontifícia Universidade Católica do Rio Grande do Sul (PUCRS), em Porto Alegre, RS, Brasil. Coordenadora Pedagógica Colégio Marista São Pedro, Porto Alegre, RS, Brasil.

\section{Endereço para correspondência}

Artur Antunes Navarro Valgas

Colégio Marista São Pedro

Rua Álvaro Chaves, 625

Floresta, 90220-040

Porto Alegre, RS, Brasil

Os textos deste artigo foram revisados pela Poá Comunicação e submetidos para validação do(s) autor(es) antes da publicação. 